\title{
Efecto del método de propagación y sitio sobre atributos fustales en árboles de Pinus radiata a la edad de 17 años
}

\section{Effect of propagation method and site on stem attributes on 17 years-old Pinus radiata trees}

\author{
Marcial Antillanca ', Guillermo Trincado ${ }^{1 *}$ y Jean Pierre Lasserre ${ }^{2}$
}

1 Universidad Austral de Chile. Facultad de Ciencias
Forestales y Recursos Naturales. Instituto de Bos-

2 Forestal Mininco S.A., Concepción, Chile.

* Autor de correspondencia. gtrincad@uach.cl ques y Sociedad. Valdivia, Chile.

\section{RESUMEN}

La tasa de plantaciones de pino radiata en Chile ha requerido aumentar la escala de producción de plantas aplicando propagación vegetativa. Sin embargo, se desconoce si existen diferencias de crecimiento y atributos fustales entre árboles reproducidos mediante semillas o estacas. Esta investigación cuantifica el efecto del método de propagación y sitio sobre atributos fustales de pino radiata a la edad de 17 años. Los atributos evaluados fueron: forma fustal, localización del centro de volumen y centro de gravedad, y ahusamiento en trozas. Los datos se obtuvieron de un ensayo silvícola sin raleo ni poda ubicado sobre suelos de ceniza volcánica y arenas. En cada sitio se establecieron dos parcelas $(30 \mathrm{~m} \times 21 \mathrm{~m})$, considerando una densidad inicial de 1428 individuos por hectárea de la misma familia genética. Dentro de cada sitio se asignó en forma aleatoria a cada parcela, plantas propagadas por semilla o por estacas de un año de edad. La combinación de métodos de propagación y sitios definieron un diseño factorial $2 \times 2$. En cada tratamiento, se seleccionaron 20 individuos para muestreo destructivo. Los resultados indican que no hubo efectos significativos de los métodos de propagación para el factor y cociente de forma, centro de volumen y gravedad. Tampoco hubo efectos significativos de los sitios ni interacción entre factores. Sin embargo, el ahusamiento de la primera troza basal (0 m - $3 \mathrm{~m}$ ) evidenció efectos significativos de ambos factores. Se puede concluir que individuos propagados por estacas y establecidos en arenas presentan trozas basales más cilíndricas que individuos establecidos en suelos derivados de cenizas volcánicas.

PALABRAS ClAVE: centro de gravedad, centro de volumen, forma fustal, modelos de efectos-mixtos, propagación por estacas, propagación por semillas.

\section{ABSTRACT}

The rate of establishment of radiata pine plantations in Chile has required increasing the production of plants propagated by vegetative methods. However, it is unknown whether there are differences in growth rates and stem attributes between trees propagated by seedlings or cuttings. This study quantifies differences between plant reproduction methods (seedling and cutting) and its interaction with site on stem attributes of radiata pine trees at the age of 17 years old. The evaluated attributes were stem form, location of center of volume and center of gravity and taper of bottom logs. The required information was obtained from an un-thinned and un-prunned trial located in two contrasting site conditions on volcanic ash and sandy soils. On each site, two rectangular plots $(30 \mathrm{~m} \times 21 \mathrm{~m})$ with initial stand density of 1428 individuals per hectare that belong to the same genetic family were established. For each plot, plants propagated by seedlings or one-year-old cuttings were randomly assigned. The combination of propagation methods and sites defined a $2 \times 2$ factorial experiment. From each treatment, twenty trees were selected for destructive sampling. The results obtained indicate that there are no significant differences between propagation methods for stem form, form quotient, location of the center of volume and center of gravity. There were also non-significant differences between sites and there was no interaction between sites and propagation methods. However, the taper of the first $\log (0 \mathrm{~m}-3 \mathrm{~m})$ was significantly different between propagation methods and between sites. We concluded that trees propagated by cuttings and established in a low productivity site on sandy soils have a more cylindrical first-bottom log in comparison to trees propagated by seedlings and established in a high productivity site on volcanic ash soils located in the Andean piedmont.

Key words: center of gravity, center of volume, tree form, mixed-effects models, propagation by cuttings, propagation by seedlings. 


\section{INTRODUCCIÓN}

La tasa promedio de establecimiento de plantaciones de Pinus radiata D. Don. en Chile durante los últimos años ha sido cercana a 50000 hectáreas anuales (Instituto Forestal [Infor], 2013). La alta demanda de plantas ha requerido aumentar su escala de producción y, por lo tanto, implementar operacionalmente procesos de propagación masiva. El proceso de producción de plantas utiliza principalmente dos métodos de reproducción: reproducción sexual y reproducción vegetativa. En el país se estima que, para P. radiata, $80 \%$ de las plantas se producen a partir de estacas provenientes de plantas mejoradas genéticamente (Corporación Chilena de la Madera, 2011; Infor, 2013). Este tipo de propagación ha tenido un auge considerable debido a la capacidad de producir mayor número de plantas con mayor uniformidad en los atributos de los individuos propagados (Holden, Klomp, Hong y Menzies, 1995). Sin embargo, diversos estudios han publicado que existen diferencias en tasas de crecimiento y atributos fustales entre árboles reproducidos mediante semillas y a través de estacas (Budianto, Tombleson y Gordon, 1998). Estas diferencias pueden tener un impacto directo en el contenido volumétrico y en la calidad de las trozas que se producen, afectando el rendimiento y aprovechamiento industrial. Diversos estudios han encontrado que individuos propagados a partir de estacas presentan un menor volumen fustal sin corteza en comparación con individuos propagados por semilla (Penman, 1988; Bundianto et al., 1998). En Chile, solamente se han realizado estudios comparativos de plantas de $P$. radiata propagadas por estacas y semillas a edades jóvenes. Vergara (2005) no encontró diferencias entre plantas propagadas por semillas y estacas de una temporada en cuanto a las tasas de crecimiento en diámetro y altura a la edad de 10 años. Sin embargo, detectó diferencias significativas en la forma del fuste medido a través de un método visual. Holden et al., (1995) tampoco detectaron diferencias en cuanto a las tasas de crecimiento entre individuos propagados por semillas y estacas de tres y seis años de edad. Contrariamente, Klomp y Hong (1985) determinaron que durante los primeros 5 años a 15 años de edad las tasas de crecimiento en altura de plantas de $P$. radiata propagadas por semilla fueron superiores a las de plantas propagadas por estacas. A pesar de que en los escasos estudios realizados, se han detectado diferencias entre plantas propagadas por distintos métodos de reproducción, no existe evidencia que estas diferencias se mantengan a la edad de rotación. Al igual que el tipo de plantas, el sitio es un factor importante a considerar durante la etapa de establecimiento de plantaciones forestales, debido a que el desarrollo y rendimiento dependerá principalmente de los factores climáticos y edáficos (Gerding y Schlatter, 1995).

En este estudio se realizó una evaluación del efecto del método de propagación y su interacción con el sitio sobre atributos fustales de $P$. radiata a la edad de 17 años. Los individuos fueron propagados por semilla y estacas de un año de edad fisiológica provenientes de la misma familia genética. Ambos tipos de individuos representan parte de las poblaciones de plantaciones comerciales que actualmente se encuentran en edad de cosecha. Los ensayos se establecieron en suelos de cenizas volcánicas y suelos de arenales, siendo sitios representativos de plantaciones localizadas en la región del Biobío. Sitios con suelos de cenizas volcánicas son más equilibrados en condiciones de menor humedad ambiental, conteniendo una alta cantidad de agua aprovechable del suelo y gran volumen de espacio arraigable en comparación con sitios con suelos de origen arenal (Ruiz, 1998). Los suelos de arenales son más limitados por su composición textural, lo que causa baja a muy baja cantidad de agua aprovechable. Además, las condiciones nutritivas son pobres debido a su bajo contenido de materia orgánica (Edwards, 1997).

\section{OBJETIVOS}

Realizar una comparación de atributos fustales entre individuos de $P$. radiata propagados por semilla y por estaca, a la edad de 17 años. Los atributos considerados en la comparación fueron: forma fustal, localización del centro de volumen y centro de gravedad fustal y ahusamiento de las primeras tres trozas basales de $3 \mathrm{~m}$ de longitud.

La hipótesis que sustenta esta investigación es que para la misma familia genética el sitio y método de propagación afectan significativamente los atributos fustales. 


\section{MATERIALES Y MÉTODOS}

\section{Sitios de estudio y material genético}

La información necesaria para este estudio se obtuvo de dos ensayos silvícolas cuyo objetivo original fue evaluar el establecimiento y crecimiento inicial de plantas de $P$. radiata. Estos ensayos fueron establecidos por Forestal Mininco S.A., durante el año 1995 en la región del BíoBío, Chile. Los ensayos están ubicados en los predios Santa Lucía que se localiza en la precordillera de Los

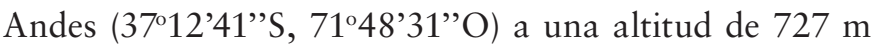
snm y Santa Isabel que se localiza en la Depresión Intermedia (37024'45's, 72'15'29'”) a una altitud de $170 \mathrm{~m}$ snm. Santa Lucía presenta una precipitación media anual que oscila entre $1400 \mathrm{~mm}$ y $2200 \mathrm{~mm}$, una temperatura media de $10,9^{\circ} \mathrm{C}$ (Schlatter, Gerding y Adriazola, 1994) y se ubica sobre un suelo de cenizas volcánicas jóvenes, correspondiente a la serie Santa Bárbara (Typic Haploxerands [Andisol]) (Ciren, 1999). Contrariamente, Santa Isabel presenta una precipitación media anual menor que oscila entre $1000 \mathrm{~mm}$ y $1450 \mathrm{~mm}$, una temperatura media de $12,9^{\circ} \mathrm{C}$ (Schlatter et al., 1994) y se ubica sobre un suelo de tipo arenal correspondiente a la serie Coreo (Typic Vitrixerands [Andisol]) (Ciren, 1999).

En cada sitio se establecieron dos parcelas de $630 \mathrm{~m}^{2}$ $(30 \mathrm{~m} \times 21 \mathrm{~m})$ y en forma aleatoria se asignaron a cada parcela plantas propagadas mediante semilla o mediante estacas (cutting). Dentro de cada parcela se establecieron seis hileras de 15 plantas con un distanciamiento de 2,0 m $\times$ 3,5 m equivalentes una densidad inicial de 1428 plantas por hectárea.

El material genético o germoplasma requerido para la propagación de plantas (semillas y estacas) establecidas en ambos sitios se obtuvo del huerto semillero clonal "Colicheu", localizado en la comuna de Cabrero, VIII región del Bío-Bío. La madre fue obtenida de plantaciones localizadas en sitios de arenas por medio de selección masal y establecida en el huerto mediante injerto. Los criterios de selección de la madre fueron el volumen y rectitud fustal. Posteriormente, la cosecha de semillas fue por medio de polinización abierta desde la madre, por lo tanto, los indi- viduos corresponden a medios hermanos. Contrariamente, el material propagado por medio de estacas se obtuvo aplicando una técnica de propagación denominada topping de primer orden, extraído al primer año de germinación de las semillas obtenidas desde la madre. Posteriormente, este material fue enraizado en contenedores de $125 \mathrm{~cm}^{3}$, teniendo como resultado una producción familiar (misma familia) (White, Adams y Neale, 2007). Por lo tanto, este material presentó un año más que el material obtenido por semillas. Esta técnica de reproducción vegetativa representa el estado del arte para la propagación de plantas utilizada en esos años y a partir de la cual se generaron parte de las plantaciones que actualmente se encuentran en edad de cosecha en las áreas de estudio.

\section{Medición de ensayos y muestreo destructivo}

Las mediciones de los individuos se realizaron en agosto de 2012 a la edad 17 años. Cabe señalar, que las parcelas no tuvieron ningún tipo de intervención de podas ni raleos previos a las mediciones, que pudieran afectar a los atributos fustales evaluados. Para cada tratamiento que se originó de la combinación de sitios y métodos de propagación $(2 \times 2)$ se seleccionaron 20 árboles tratando de cubrir dentro de cada parcela todo el conjunto de clases diamétricas; completando una muestra total de 80 individuos (Tabla 1). Una vez derribados, cada individuo fue desramado para realizar las mediciones de perfiles fustales y el registro de las variables de estado: altura de tocón, diámetro a la altura de pecho (DAP) y altura total $(\mathrm{H})$. La primera medición de diámetro fustal se realizó en la base del árbol, una segunda medición fue equidistante entre la base y el DAP, una tercera medición en el DAP; desde este punto todas las mediciones posteriores se realizaron cada $1 \mathrm{~m}$ ( $2 \mathrm{~m}, 3 \mathrm{~m}, 4 \mathrm{~m}$, etc.) hasta alcanzar un diámetro fustal igual a $10 \mathrm{~cm}$. A partir de estas mediciones se calculó el volumen fustal sin corteza (Vssc) aplicando la fórmula de Smalian (Avery y Burkhart, 2002).

Para la estimación de la densidad de madera en condición verde (densidad verde, calculada como peso verde/ volumen verde) a lo largo del fuste se extrajeron rodelas en 
TAвla 1. Estadística descriptiva de individuos seleccionados para cada tratamiento $(\mathrm{n}=20)$.

\begin{tabular}{|c|c|c|c|c|}
\hline \multirow[t]{2}{*}{ Variables } & \multicolumn{2}{|c|}{ Individuos de semilla } & \multicolumn{2}{|c|}{ Individuos de estacas } \\
\hline & Media & Intervalo & Media & Intervalo \\
\hline \multicolumn{5}{|c|}{ Suelo de cenizas volcánicas } \\
\hline $\mathrm{DAP}(\mathrm{cm})$ & 26,4 & $18,9-33,4$ & 30,1 & $21,6-40,1$ \\
\hline $\mathrm{H}(\mathrm{m})$ & 27,3 & $21,3-31,6$ & 29,6 & $26,1-33,3$ \\
\hline Vssc $\left(\mathrm{m}^{3}\right.$ árbol $\left.^{-1}\right)$ & 0,63 & $0,23-1,10$ & 0,81 & $0,32-1,50$ \\
\hline \multicolumn{5}{|c|}{ Suelo arenales } \\
\hline $\mathrm{DAP}(\mathrm{cm})$ & 26,8 & $19,7-32,2$ & 26,9 & $18,5-34,2$ \\
\hline$H(m)$ & 26,3 & $22,8-28,4$ & 25,8 & $23,1-27,7$ \\
\hline Vssc $\left(\mathrm{m}^{3}\right.$ árbol $\left.^{-1}\right)$ & 0,52 & $0,20-0,90$ & 0,53 & $0,20-0,90$ \\
\hline
\end{tabular}

la siguiente secuencia: base, DAP, $3 \mathrm{~m}, 6 \mathrm{~m}, 9 \mathrm{~m}, 12 \mathrm{~m}, 15$ m e índice de utilización $10 \mathrm{~cm}$. Cada rodela fue cubierta con el fin de evitar la pérdida de humedad de la madera y fueron transportadas al laboratorio para determinación de densidad verde.

\section{Estimación de forma fustal}

La estimación de la forma fustal para cada individuo se realizó calculando el factor de forma natural:

$$
f_{0,1}=\frac{\mathrm{V}}{\mathrm{V}_{0,1 H}}
$$

donde $f_{0,1}$ es el factor de forma natural, $\mathrm{V}$ es el volumen fustal IU=10 $\mathrm{cm}$ sin corteza $\left(\mathrm{m}^{3} \mathrm{ssc}\right), \mathrm{V}_{0,1 \mathrm{H}}$ es el volumen del cilindro $\left(\mathrm{m}^{3} \mathrm{ssc}\right)$, calculado con un diámetro sin corteza medido a un décimo de la altura total del fuste. Adicionalmente, se determinó el cociente de forma natural (Prodan, Peters, Cox y Real, 1997; Van Laar y Akca, 2007):

$q_{0,5}=\frac{\mathrm{d}_{H / 2}}{\mathrm{D}_{0,1 H}}$

donde $q_{0,5}$ es el cociente de forma natural, $\mathrm{d}_{\mathrm{H} / 2}$ corresponde al diámetro sin corteza $(\mathrm{cm})$ medido en la mitad del largo fustal y $\mathrm{D}_{0,1 \mathrm{H}}$ es el diámetro sin corteza $(\mathrm{cm})$ medido a un décimo de la altura total.

\section{Estimación del centro de volumen fustal}

La localización del centro de volumen (CV) fustal está definido como la altura donde se encuentra $50 \%$ del volumen total del fuste (MacDonald y Forslund, 1986). Para su determinación se empleó un método de aproximación numérica: 1) se calculó el volumen total del fuste sin corteza a través de la acumulación de volúmenes de las secciones de un metro de longitud utilizando la fórmula de Smalian; 2) se realizó una acumulación de volúmenes de secciones hasta que el volumen acumulado sea mayor al volumen medio del fuste y 3) finalmente, se interpoló la altura que divide el volumen fustal en dos partes iguales, siendo ese punto la altura fustal correspondiente a la localización del centro de volumen $(\mathrm{CV})$.

\section{Estimación del centro de gravedad fustal}

El centro de gravedad (CG) se define como el punto en el que se encuentra aplicada la resultante de todas las fuerzas gravitacionales de un cuerpo (Gettys, Keller y Skove, 1991). Se calcula como la razón entre el primer momento de masas $^{1}(\mathrm{M})$ y la masa total (W) de un cuerpo. La loca-

1 El primer momento de masas corresponde a la distribución de masas de cada sección del fuste respecto a un eje de rotación horizontal (Hewitt, 2004). 
lización del centro de gravedad a lo largo del fuste ha sido de utilidad para diseñar máquinas procesadoras que eviten el quiebre de los fustes al momento del trozado (Corona, 1992). Para su estimación se utilizó la siguiente fórmula:

$G=\frac{\mathrm{M}}{\mathrm{W}}=\frac{\sum_{i=1}^{n \mathrm{sec}} \mathrm{M}_{i}}{\sum_{i=1}^{n \mathrm{sec}} \mathrm{W}_{i}}$

donde $\mathrm{M}_{\mathrm{i}}$ corresponde al primer momento de la $i$-ésima sección fustal y $\mathrm{W}_{\mathrm{i}}$ es la masa de la $i$-ésima sección fustal que se calcula multiplicando el volumen de la sección por la densidad verde de la madera. El procedimiento utilizado es equivalente a la aplicación de cálculo integral presentado por Lynch, Wiant y Patterson, (1994). A partir de la medición de las secciones fustales se utilizaron las siguientes fórmulas de aproximación numérica:

$$
\begin{aligned}
& \mathrm{M}=\frac{\pi}{40.000} \cdot \sum_{i=1}^{n \sec }\left(\frac{\mathrm{di}_{i}^{2} \cdot \mathrm{hi}_{i}+\mathrm{ds}_{i}^{2} \cdot \mathrm{hs}_{i}}{2} \cdot \mathrm{DV}_{i} \cdot \mathrm{L}_{i}\right) \\
& \mathrm{W}=\frac{\pi}{40.000} \cdot \sum_{i=1}^{n \sec }\left(\frac{\mathrm{di}_{i}^{2}+\mathrm{ds}_{i}^{2}}{2} \cdot \mathrm{DV}_{i} \cdot \mathrm{L}_{i}\right)
\end{aligned}
$$

donde, $\mathrm{ds}_{\mathrm{i}}$ corresponde al diámetro superior $(\mathrm{cm}) \sin$ corteza de la $i$-ésima sección, di $_{i}$ es el diámetro inferior (cm) sin corteza de la $i$-ésima sección, hi $i_{\mathrm{i}}$ altura fustal (m) de la base de la $i$-ésima sección, hs $\mathrm{s}_{\mathrm{i}}$ altura fustal (m) superior de la $i$-ésima sección, $\mathrm{L}_{\mathrm{i}}$ largo de la $i$-ésima sección (m) y $\mathrm{DV}_{\mathrm{i}}$ es densidad verde de la sección $\left(\mathrm{kg} \mathrm{m}^{-3}\right)$.

Para efecto de comparación entre individuos, se determinó la posición del centro de gravedad (CG) a lo largo del fuste en términos relativos. La aplicación de las fórmulas anteriores requirió la construcción de un modelo predictor de densidad verde. El modelo utilizado para representar el comportamiento de la densidad verde a lo largo del fuste fue un polinomio cúbico:

$$
\mathrm{DV}_{h}=\beta_{0}+\beta_{1}\left(\frac{\mathrm{h}}{\mathrm{H}}\right)+\beta_{2}\left(\frac{\mathrm{h}}{\mathrm{H}}\right)^{2}+\beta_{3}\left(\frac{\mathrm{h}}{\mathrm{H}}\right)^{3}
$$

donde $\mathrm{DV}_{b}$ corresponde a la densidad verde observada $\left(\mathrm{kg} \mathrm{m}^{-3}\right)$ a la altura fustal h $(\mathrm{m}), \mathrm{H}$ es la altura total fustal del árbol (m) y $\beta_{\mathrm{i}}$ corresponde a los parámetros fijos a ser estimados del modelo $(i=0, \ldots, 3)$. Watt y Trincado (2014) utilizaron el mismo procedimiento para modelar el comportamiento de la densidad verde a lo largo del fuste para $P$. radiata. Con el propósito de modelar el comportamiento de densidad verde a lo largo del fuste para cada individuo, la estimación de parámetros se realizó para cada tratamiento aplicando técnicas de modelamiento lineal de efectos mixtos (Verbeke y Molenberghs, 1997). La figura 1 presenta el ajuste de la curva del modelo a los datos de cada tratamiento.

Se aprecia que el comportamiento de la densidad a lo largo del fuste es similar para los cuatro tratamientos y que puede ser modelada por el polinomio cúbico. La densidad verde es mayor en la base del fuste produciéndose una disminución a una altura relativa de $20 \%$ y luego aumenta para disminuir en la parte superior del fuste. El modelo desarrollado permitió predecir para cada individuo valores de densidad verde en los extremos de cada sección medida y el promedio de ambas densidades se utilizó para evaluar las ecuaciones (4) y (5). Para la estimación de parámetros se utilizó la rutina Proc Mixed contenida en el software estadístico Statistical Analysis System SAS 9.2 (SAS Institute, 1999). Los parámetros fijos estimados y componentes de varianza del modelo utilizado para los cuatro tratamientos fueron altamente significativos $(P<0,0001)$. Para cada árbol se predijo la densidad verde a lo largo del fuste en los puntos de medición del perfil fustal utilizando la respuesta calibrada. Esto permitió posteriormente calcular la posición relativa del centro de gravedad a lo largo del fuste utilizando la fórmula (4). La figura 2 presenta la respuesta media y calibrada de densidad verde a lo largo del fuste para un árbol muestra localizado en Sta. Lucía (suelo cenizas volcánicas) y propagado por estaca (Árbol $=16, \mathrm{DAP}=27,5 \mathrm{~cm}$ y $\mathrm{H}=28,2 \mathrm{~m})$. Se aprecia que la respuesta calibrada modela de mejor manera la tendencia de los datos observados para este individuo que la respuesta media (Fig. 2). 

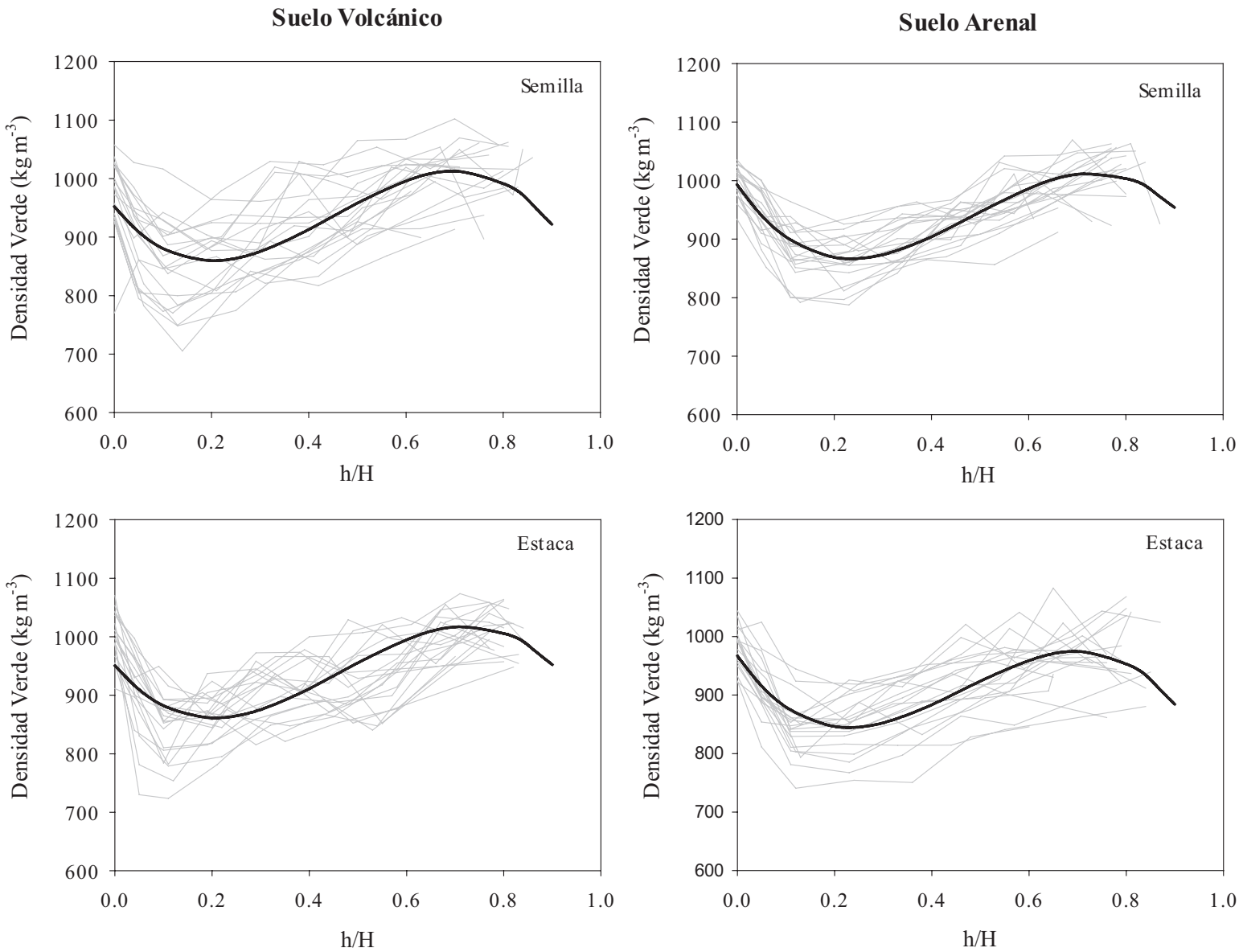

Figura 1. Variación de la densidad verde a lo largo del fuste para los cuatro tratamientos.

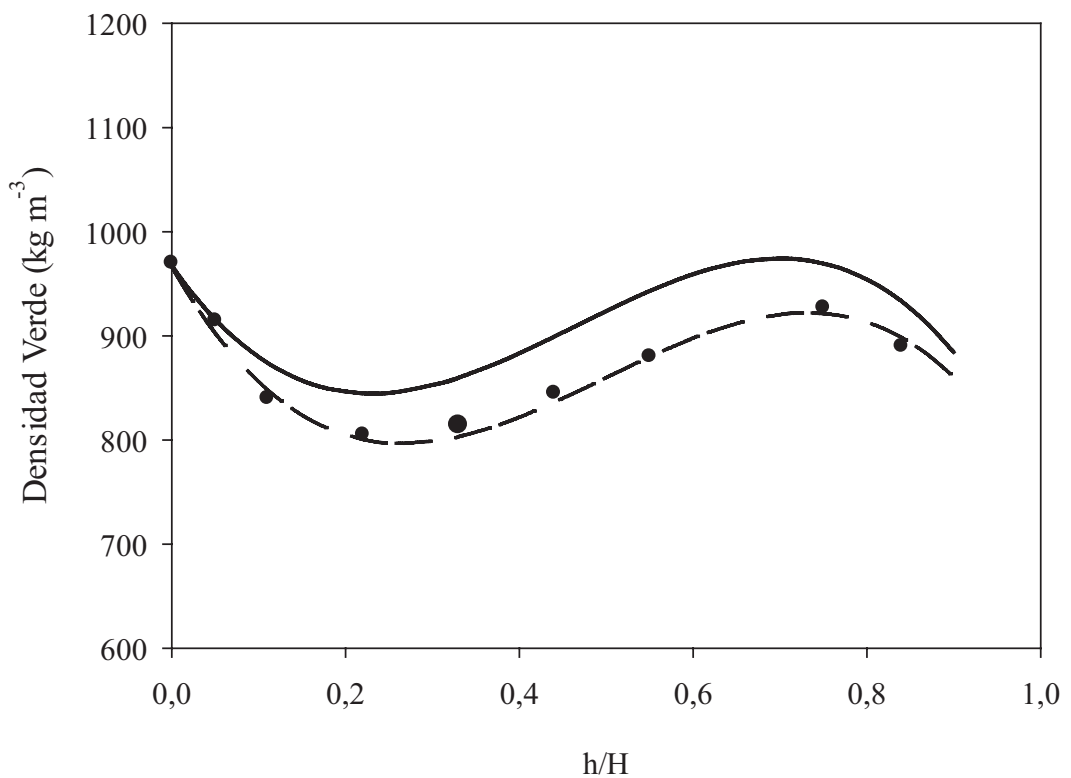

Figura 2. Curva de densidad verde para la respuesta media (línea continua) y respuesta calibrada (línea segmentada) respecto a valores observados $(\bullet)$ a lo largo del fuste. 
La estimación del ahusamiento para cada una de las tres primeras trozas basales $(0 \mathrm{~m}-3 \mathrm{~m}, 3 \mathrm{~m}-6 \mathrm{~m}$ y $6 \mathrm{~m}-9$ $\mathrm{m})$ se calculó como la diferencia entre el diámetro basal y el diámetro menor de la troza dividida por el largo de la sección (3 m).

\section{Análisis estadístico}

Para la comparación entre tratamientos se aplicó un análisis de varianza (Andeva) de tipo factorial $2 \times 2$. Los factores de interés fueron el método de propagación (estaca y semilla) y el sitio (arenas y cenizas volcánicas). El modelo estadístico utilizado corresponde a:

$$
Y_{i j k}=\mu+\tau_{i}+\beta_{j}+(\tau \beta)_{i j}+\varepsilon_{i j k}
$$

donde $Y_{\mathrm{ijk}}$ es la $k$-ésima observación de la variable de interés localizada en el $j$-ésimo sitio para el $i$-ésimo método de propagación, $\mu$ es la media global, $\tau_{\mathrm{i}}$ es el efecto del $i$-ésimo nivel del método de propagación, $\beta_{\mathrm{j}}$ es el efecto del $j$-ésimo nivel del factor sitio, $\tau \beta$ la interacción entre los factores y $\varepsilon$ corresponde al error aleatorio. A nivel de fuste, las variables de interés evaluadas fueron: factor de forma natural, cociente de forma natural y localización relativa del centro de volumen y centro de gravedad a lo largo del fuste. Por otro lado, a nivel de trozas, la variable respuesta fue el ahusamiento de las trozas basales. Para todas las variables de interés, las comparaciones se realizaron sin corteza considerando un nivel de significancia de $P=0,05$. Para la evaluación de las pruebas estadísticas se utilizó la rutina PROC GLM contenida en el software estadístico Statistical Analysis System SAS 9.2 (SAS Institute, 1999).

\section{RESULTADOS}

Los datos de atributos fustales cumplieron con los supuestos de normalidad (prueba de normalidad) y homocedasticidad de varianza (prueba de Levene) siendo en ambos casos no significativo $(P>0,05)$. Por lo tanto, fue posible aplicar los procedimientos estadísticos paramétricos propuestos para el análisis de datos.

\section{Comparación de forma fustal}

Para el factor de forma natural y cociente de forma no se observaron efectos significativos del método de propagación (P), ni tampoco del sitio (S). De la misma forma la interacción $(\mathrm{S} \times \mathrm{P})$ resultó ser significativa (Tabla 2). El factor de forma natural presentó valores medios entre 0,51 - 0,53 y el cociente de forma natural presentó valores medios que fluctuaron entre 0,70 - 0,72. Los datos mostraron como tendencia que el factor de forma natural y cociente de forma de individuos proveniente de estacas fueron levemente mayores en comparación con individuos propagados por semilla (Tabla 2).

\section{Localización centro de volumen y centro de gravedad}

Para el centro de volumen y centro de gravedad tampoco hubo efecto del método de propagación (P) ni del sitio (S), no hubo interacción significativa entre ambos factores (Tabla 2). Los valores de localización del centro de volumen relativo a lo largo del fuste se encontraron entre 0,20 - 0,22 (Tabla 2). Esto indicaría que 50\% del volumen total del fuste se encuentra entre $20 \%-22 \%$ de la altura total del árbol. Sin embargo, se aprecia una tendencia: la localización del centro de volumen en árboles propagados por estacas se encuentra a una mayor altura que en árboles propagados por semilla (Tabla 2). El centro de gravedad relativo presentó un valor promedio de 0,31 para ambos sitios y para ambos métodos de propagación (Tabla 2).

\section{Ahusamiento de trozas basales}

Únicamente para la primera troza basal se detectaron diferencias significativas de ahusamiento entre métodos de propagación y entre sitios (Tabla 2). El ahusamiento de la primera troza basal $(0 \mathrm{~m}-3 \mathrm{~m})$ presentó valores promedio entre $1,95 \mathrm{~cm} \mathrm{~m}^{-1}$ y $2,73 \mathrm{~cm} \mathrm{~m}^{-1}$. Esto sugiere que la primera troza basal $(0 \mathrm{~m}-3 \mathrm{~m})$ proveniente de individuos propagados por semilla presenta significativamente mayor ahusamiento que la primera troza basal proveniente de estaca (Fig. 3). Por otro lado, individuos localizados en el sitio con suelo volcánico presentaron, también para la primera troza basal, un ahusamiento significativamente 
Tавца 2. Valores medios de atributos fustales medidos en árboles muestra.

\begin{tabular}{|c|c|c|c|c|c|c|c|}
\hline \multirow[t]{2}{*}{ Atributos de interés } & \multicolumn{2}{|c|}{ Suelo de cenizas volcá-nicas } & \multicolumn{2}{|c|}{ Suelo arenales } & \multicolumn{3}{|c|}{ Análisis de varianza (Andeva) } \\
\hline & Semilla & Estaca & Semilla & Estaca & Propagación (P) & Sitio (S) & $P \times S$ \\
\hline \multicolumn{8}{|l|}{ Nivel fustal } \\
\hline Factor de forma natural $f_{0.1}$ & 0,51 & 0,52 & 0,52 & 0,53 & $0,90^{\text {ns }}$ & 0,30 ns & $0,22^{\text {ns }}$ \\
\hline Cociente de forma natural $q_{0,5}$ & 0,70 & 0,71 & 0,71 & 0,72 & $0,85^{\text {ns }}$ & $0,45^{\text {ns }}$ & $0,40^{\text {ns }}$ \\
\hline Centro de volumen relativo & 0,21 & 0,22 & 0,20 & 0,22 & $0,58^{\text {ns }}$ & $0,44^{\mathrm{ns}}$ & $0,15^{\text {ns }}$ \\
\hline Centro de gravedad relativo & 0,31 & 0,31 & 0,31 & 0,31 & $0,32^{\text {ns }}$ & $0,12^{\text {ns }}$ & $0,65^{\text {ns }}$ \\
\hline \multicolumn{8}{|l|}{ Nivel de trozas (ahusamiento $\mathrm{cm} \mathrm{m}^{-1}$ ) } \\
\hline Troza 1 (O m - 3 m) & 2,73 & 2,41 & 2,28 & 1,95 & $0,026^{*}$ & $0,002^{*}$ & $0,965^{\mathrm{ns}}$ \\
\hline Troza 2 (3 m - 6 m) & 0,75 & 0,57 & 0,66 & 0,62 & $0,089^{\text {ns }}$ & $0,796^{\text {ns }}$ & $0,251^{\text {ns }}$ \\
\hline Troza 3 (6 m - 9 m) & 0,54 & 0,63 & 0,56 & 0,55 & $0,343^{\text {ns }}$ & $0,397^{\mathrm{ns}}$ & $0,193^{\text {ns }}$ \\
\hline
\end{tabular}

ns: no significativo para $\mathrm{P}=0,05 *: P<0,05$.

mayor que aquellos individuos localizados en el sitio arenal (Fig. 3). El mayor ahusamiento de la primera troza basal implica que las trozas son más cónicas y presentarán un menor rendimiento industrial para la producción de madera aserrada en comparación a trozas más cilíndricas.

\section{DISCUSIÓN}

Para $P$. radiata se han encontrado diferencias significativas en la forma del fuste cuando se han comparado individuos propagados con distintos métodos de reproducción (Arnold y Gleed, 1985; Vergara, 2005). Budianto et al., (1998) compararon para esta especie la forma fustal de individuos de estacas y semilla a la edad de 26 años y encontraron que plantas provenientes de estacas presentaron fustes más cilíndricos. En este estudio, en cambio, no se encontraron diferencias significativas entre individuos propagados por semilla y estacas respecto al factor y cociente de forma natural. Estos resultados se podrían explicar por la edad fisiológica de la estaca utilizada para la reproducción de plantas. Para este estudio las plantas fueron propagadas a partir de estacas de primer orden que tenían apenas un año de edad. Existen estudios que indican que, a mayor edad fisiológica de las estacas, mayores son las diferencias con plantas propagadas por semillas en relación con la capacidad de enraizamiento, tasas de crecimiento, espesor de corteza y forma fustal (Holden, Klomp, Hong y Menzies, 1995). Menzies, Faulds, Holden, Kumar y Klomp (2004) comparó, para P. radiata a la edad de 12 años, el comportamiento de estacas considerando distintas edades fisiológicas (1 año a 5 años). Ellos indican que el uso de estacas de 3 años mejora la forma fustal pero sin que exista una pérdida de crecimiento a edades tempranas en comparación con plantas propagadas por semillas.

Con respecto a forma fustal tampoco se detectaron diferencias significativas entre sitios localizados en suelos de ceniza volcánica y suelos arenales. Se esperaría que árboles creciendo en arenas presentaran menor ahusamiento que árboles creciendo en suelos de ceniza volcánica debido a su menor productividad. Sin embargo, la condición de sitio corresponde a arenas húmedas que presenta una productividad media a alta para $P$. radiata. Otro factor que podría explicar que no existan diferencias entre sitios es la alta densidad de las plantaciones en los dos estudiados, debido a no haberse realizado intervenciones de podas y raleos.

Tal como se indicó anteriormente, no hubo efectos significativos del método de propagación y sitio sobre la 

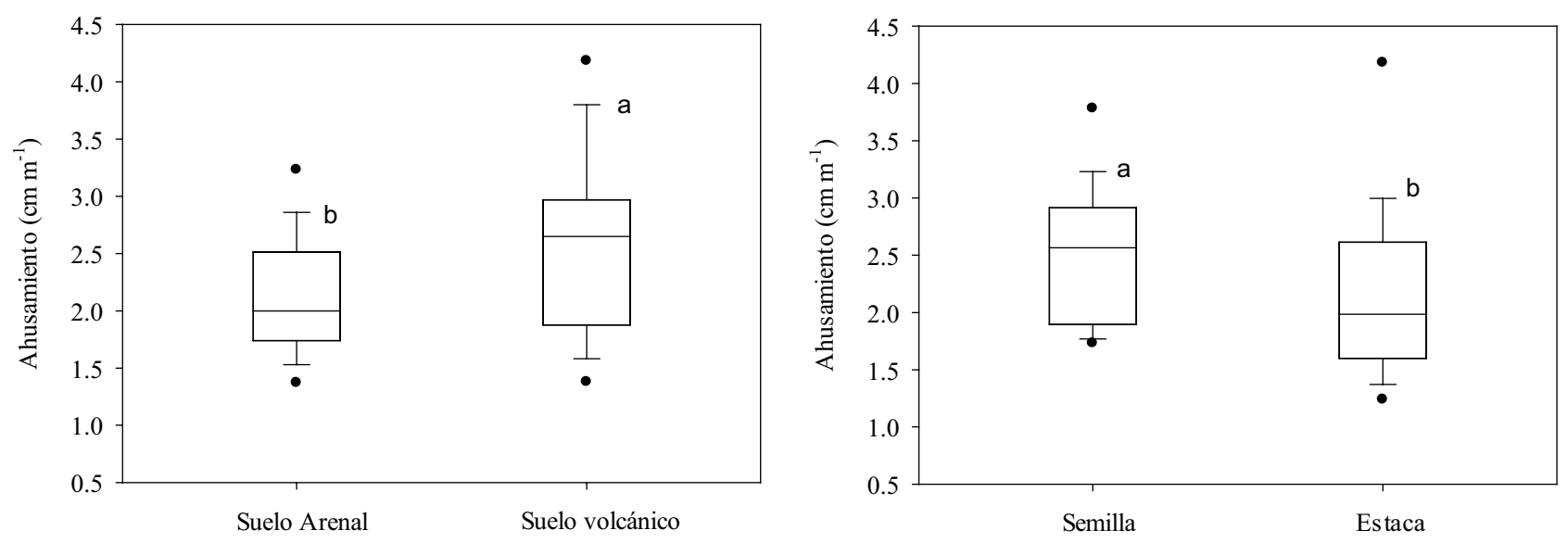

Figura 3. Comparación del ahusamiento para la primera troza basal entre métodos de propagación y entre sitios.

localización del centro de volumen y gravedad de los árboles. El centro de volumen relativo se localizó para los distintos tratamientos entre 0,20-0,22 de la altura fustal; correspondiente a una altura fustal de $5,3 \mathrm{~m}-5,5 \mathrm{~m}$ (Tabla 2). Para el centro de gravedad se determinó un valor promedio de 0,31 (Tabla 2). Corona (1993) encontró valores entre 0,29 a 0,34 para $P$. radiata. Estos valores son similares a los publicados para otras especies. Forslund (1982) determinó que el centro de gravedad para Populus spp se encontraba a una altura relativa de 0,3 y Fridley y Tufts (1989) mencionan valores entre 0,36 y 0,39 para $P$. taeda. La determinación del centro de gravedad ha sido utilizada para la construcción de funciones de volumen (MacDonald y Forslund, 1986) y para desarrollar estudios biomecánicos de estabilidad del fuste necesarios para mejorar equipos y procesos durante la cosecha mecanizada (Lynch, Max, Burkhart y Liu, 1993).

Cabe señalar que en este estudio se presenta un procedimiento para predecir densidad verde a lo largo del fuste aplicando técnicas de modelamiento lineal de efectos mixtos. El procedimiento demostró ser de utilidad para predecir densidad verde a distintas alturas fustales que fue requerida para estimar la posición del centro de gravedad para cada uno de los árboles.

En este estudio se determinó que existe un efecto significativo del método de propagación y sitio respecto al ahusamiento de la primera troza basal (0 m - $3 \mathrm{~m})$. Se ha publicado que existe un mejor aprovechamiento en productos como chapa y madera aserrada desde trozas provenientes de estacas debido a que son más cilíndricas que aquellas provenientes de semillas (Spencer, 1987; Holden et al., 1995). Estos resultados son coincidentes con el presente estudio, en el que se determinó que las trozas basales más cilíndricas se producirían a partir de plantas propagadas por estacas y que crecen en suelos arenales.

\section{CONCLUSIONES}

Para el mismo material genético no se detectaron diferencias significativas entre métodos de propagación y entre sitios respecto a la forma fustal y centro de volumen y gravedad de los árboles a la edad de 17 años. Un factor que puede explicar los resultados obtenidos es la edad fisiológica de las estacas utilizadas en este estudio. En este caso, las estacas fueron obtenidas al primer año de germinación de las plantas. Por lo tanto, no existe una diferencia importante en relación a edad fisiológica con las plantas reproducidas por semilla. Según lo encontrado en la literatura, se esperarían mayores diferencias respecto a atributos fustales entre métodos de propagación al utilizar estacas de 2 años de edad o mayores. Sin embargo, individuos propagados por estacas y establecidos en arenas presentaron trozas basales $(0 \mathrm{~m}-3 \mathrm{~m})$ significativamente más cilíndricas o con un menor ahusamiento que individuos establecidos en la precordillera de Los Andes en sitios de 
ceniza volcánica. Esto indicaría que los mayores efectos de método de propagación y sitio se pueden detectar en la base fustal de los árboles.

\section{RECONOCIMIENTOS}

Los autores agradecen al Fondo Nacional de Desarrollo Científico y Tecnológico (FONDECYT) por el financiamiento de esta investigación a través del proyecto $\mathrm{N}^{\circ}$ 1120433 "Evaluating and Modeling the impact of environmental conditions on radiata pine wood and fibre properties" y a Forestal Mininco S.A. por proveer con los datos necesarios y apoyo logístico de campo.

\section{REFERENCIAS}

Arnold, R. y Gleed, J. (1985). Raising and managing radiata pine cutting for production forests. Australian Forestry, 48(3), 199-206.

Avery, Th. E. y Burkhart, H. E. (2002). Forest measurements. EUA: McGraw-Hill.

Budianto, M., Tombleson, J. D. y Gordon, A. D. (1998). Comparison of taper, volume and bark thickness of 26 years old seedling and cutting from Mamaku Forest (Technical Report $\mathrm{N}^{\circ}$ 49). New Zealand: Forest Research Institute.

Centro de Información de Recursos Naturales. (1999). Estudio agrológico VIII región (Serie Técnica No 121). Santiago, Chile: CIREN.

Corona, P. (1992). An Approach to Predicting Static Properties of tree Stems. Journal of Forest Engineering, 3(2), 7-11.

Corporación Chilena de la Madera. (2011). Recuperado de http://www.corma.cl/corma.asp?id=3\&ids=535

Edwards, A. (1997). Características y variabilidad de arenales de la VIII Región, plantados con Pinus radiata D. Don. Tesis de licenciatura no publicada, Universidad de Austral de Chile, Valdivia, Chile.

Fridley, J. y Tufts, R. (1989). Analytical estimates of Loblolly Pine tree center of mass and mass moment of inertia. Forest Science, 35(1), 126-136.

Forslund, R. (1982). A geometrical tree volume model based on the location of the centre of gravity of the bole. Canadian Journal of Forest Research, 12(2), 215-221.
Gerding, V. y Schlatter, J. (1995). Variables y factores del sitio de importancia para la productividad de Pinus radiata D. Don en Chile. Bosque, 16(2), 39-56.

Gettys, W. E., Keller, F. J. y Skove, M. J. (1991). Física clásica y moderna. España: McGraw-Hill.

Hewitt, P.G. (2004). Física conceptual (9ª ed.). México: Pearson Educación de México.

Holden, D. G., Klomp, B. K., Hong, S. O. y Menzies, M. I. (1995). Growth and predicted timber value of Pinus radiata cutting and seedling on a fertile farm site. New Zealand Journal of Forestry Science, 25(3), 283-300.

Instituto forestal [INFOR]. (2013). Estadísticas forestales. Recuperado de http://wef.infor.cl/sector_forestal/sectorforestal.php\#/0

Klomp, B. K. y Hong, S. O. (1985). Performance of Pinus radiata seedlings and cuttings to age 15 years. New Zealand Journal of Forestry Science, 15(3), 281-297.

MacDonald, G. y Forslund, R. (1986). Application of a geometrical volume equation to species with different bole forms. Canadian Journal of Forest Research, 16(2), 311-314.

Menzies, M. I., Faulds, T., Holden, D. G., Kumar, S. y Klomp, B. K. (2004). Maturation status and genetic improvement effects on growth, form, and wood properties of Pinus radiata cuttings up to age 12 years. New Zealand Journal of Forestry Science, 34(3), 255-271.

Lynch, T. B., Max, T. A., Burkhart, H. E. y Liu, J. (1993). Prediction equations for centers of gravity and moments of inertia of Loblolly pine stems. Forest Science, 39(2), 260274.

Lynch, T. B., Wiant, H. V. Jr. y Patterson, D. W. (1994). Comparison of $\log$ volume estimated using formulae for $\log$ center of gravity and center of volume. Canadian Journal of Forest Research, 24(1), 133-137.

Penman, J. T. D. (1988). Volume, taper, and bark thichness in seedlings and cuttings from Mamaku forest, New Zealand. New Zealand Journal of Forestry Science, 18(3), 311-317.

Prodan, M., Peters, R., Cox, F. y Real, P. (1997). Mensura Forestal. San José, Costa Rica: IICA.

Ruiz, M. (1998). Características y variabilidad de sitios con plantaciones de Pinus radiata (D. Don) en suelos de ceni- 
zas volcánicas de las regiones VII y IX. Tesis de pregrado, Facultad de Ciencias Forestales y Recursos Naturales, Universidad de Austral de Chile, Valdivia, Chile.

Schlatter, J., Gerding, V. y Adriazola, J. (1994). Sistema de ordenamiento de la tierra, herramienta para la planificación forestal aplicado a las Regiones VII, VIII y IX (Serie Técnica). Valdivia, Chile: Facultad de Ciencias Forestales y Recursos Naturales, Universidad Austral de Chile.

SAS Institute Inc. (1999). SAS/STAT User's Guide: (Version 9.2). Cary, NC, EUA: SAS Institute.

Spencer, D. J. (1987). Increased yield of high quality veneer and sawn timber from cutting of radiata pine. Australian Forestry, 50(2), 112-117.

Van Laar, A. y Akca, A. (2007). Forest mensuration. Dordrecht, Netherlands: Springer.

Verbeke, G. y Molenberghs, G. (1997). Linear mixed models in practice: A SAS-Oriented approach. E.U.A: Springer.

Vergara, M. (2005). Evaluación de dos modelos silvopastorales efectuados con dos tratamientos de preparación de sitio y dos tipos de plantas de Pinus radiata en el predio Huape Tres Esteros. Tesis de pregrado, Universidad de Austral de Chile, Valdivia, Chile.
Watt, M. S. y Trincado, G. (2014). Modelling between tree and longitudinal variation in green density within Pinus radiata: implications for estimation of MOE by acoustic methods. New Zealand Journal of Forestry Science, 44, 1-16.

White, T. L., Adams, W. T. y Neale, D. B. (2007). Forest genetics. United Kingdom: CAB International.

Manuscrito recibido el 30 de octubre de 2015.

Aceptado el 6 de enero de 2017.

Este documento se debe citar como:

Antillanca, M., Trincado, G. y Lasserre, J. P. 2017. Efecto del método de propagación y sitio sobre atributos fustales en árboles de Pinus radiata a la edad de 17 años. Madera y Bosques, 23(1), 79-89. doi: 10.21829/myb.2017.2311554 